\title{
Environmental sustainability in supply chains
}

\author{
Jan C. Fransoo • Hans-Otto Günther • \\ Werner Jammernegg
}

Published online: 18 December 2013

(C) Springer Science+Business Media New York 2013

In almost all industries companies are confronted with the sustainability challenge induced by climate change, end of fossil fuel and scarcity of natural resources, just to name a few. In general, sustainability management-the so-called triple bottom line-seeks to balance the economic, social and environmental performance (e.g. $\mathrm{CO}_{2}$, waste, or resource consumption). Traditionally, the development of supply chains is driven by measures like costs and customer service. More recently, in view of the sustainability challenge environmental issues are increasingly taken into consideration, for instance, by adding environmental constraints to supply chain models or by considering carbon taxes and emission trading schemes or by including the effect of green house gas emissions in the choice of the transportation mode. This naturally leads to multi-objective considerations in order to analyze the trade-off between economic and environmental performance measures.

The primary objective of this special issue is to reflect the recent developments in sustainability management in supply chains and related transport systems and to examine research issues concerned with quantitative and other modeling techniques to improve the environmental performance of supply chains in industry. For this special issue twelve papers have been selected for publication after a thorough peerreview according to the standards of the FSM journal.

\section{J. C. Fransoo}

School of Industrial Engineering, Eindhoven University of Technology, P. O. Box 513, 5600 MB Eindhoven, The Netherlands

e-mail: j.c.fransoo@tue.nl

H.-O. Günther $(\bowtie)$

Department of Industrial Engineering, Seoul National University, Seoul 151-744, Korea e-mail: hans-otto.guenther@ hotmail.de

W. Jammernegg

Department of Information Systems and Operations, WU Vienna, Welthandelsplatz 1, 1020 Vienna, Austria

e-mail: werner.jammernegg@wu.ac.at 


\section{Papers in the special issue}

The first paper by K. Kim, B. Jeong and H. Jung addresses supply profitability taking environmental and societal responsibilities into account. The authors start with reviewing the development of conventional to sustainable supply chains and present a related research framework. As a starting point to analyze sustainable supply chains quantitatively they present a theoretical model which provides insight into the possible surplus to companies resulting from the transition of conventional to sustainable supply chains.

The subsequent paper by M. Kannegiesser and H.-O. Günther presents a sustainability optimization framework for strategic network design under consideration of economic, social as well as ecologic objectives. The core of the framework is an end-to-end closed-loop value chain model consisting of process, transport and product-in-use modules. The latter allows to model the sustainability of technical products during their entire lifecycle. Finally, minimize-time-tosustainability is proposed as a novel approach to minimize the time an industry supply chain needs to transform into a predefined sustainability steady state.

In the second part of the paper by M. Kannegiesser, H.-O. Günther and $O$. Gylfason the optimization framework is applied to the European automotive industry supply chain. Numerical experiments based on empirical industry data show the impact of optimization strategies on overall costs and emissions and the possible long-term development of the industry supply chain including the relocation of production capacities, the choice of transportation modes and the potential change towards lower emission products such as electric vehicles.

In his paper S.-P. Chuang develops a compound approach with a five-phase process to assess and improve a product's green performance. Based on the Six Sigma approach, a five-phase process is proposed which utilizes the analytical hierarchy process (AHP) and particle swarm optimization (PSO) to arrange the actions for accessing and improving the green performance. A case study involving two global footwear manufacturers demonstrates the effectiveness of the proposed approach.

The paper by S. Pan, E. Ballot, F. Fontane and D. Hakimi is concerned with pooling of supply chains as an emergent strategy for improving logistical performance. In a pooled supply chain, different companies share transport and logistics resources in order to improve logistics performance. As a case study the authors consider small and medium-sized food suppliers in France serving the same retail chain. Using supply network optimization models, different scenarios were investigated showing the possible savings in costs as well as $\mathrm{CO}_{2}$ emissions.

In many countries plastic recycling is a legal requirement and its environmental benefits are well known. The purpose of the paper by X. Bing, J. M. BloemhofRuwaard and J. G. A. J. van der Vorst is to provide decision support on choosing the most suitable combination of separation methods in the Netherlands. A mixedinteger linear programming (MILP) model, which minimizes both transportation cost and environmental impact, is used in this network design. The numerical study shows that the current network settings are efficient in logistics terms but have a potential to adapt to strategic changes in the design of the collection network. 
The paper by S. Treitl, P.C. Nolz and W. Jammernegg focuses on the analysis of transport processes and shows the economic and environmental effects of routing decisions in a supply chain with vertical collaboration, for instance through vendormanaged inventory. The authors propose an Inventory Routing Model and apply it to a case study from the petrochemical industry. Results show the importance of detailed transport process analyses and suggest a potential for achieving Paretoimprovements by reducing both costs and carbon emissions in a supply chain with vertical collaboration.

The effect of carbon emission regulations on transport mode selection is addressed in the paper by K. M. R. Hoen, T. Tan, J.C. Fransoo and G.J. van Houtum. They assume a 'carbon-aware' company that is reconsidering the transport mode selection decision. Traditionally the trade-off has been between lead time and transportation costs. For considering carbon emission costs they use a specific measurement methodology based on real-life data and incorporate it into an inventory model. The study indicates that transport mode selections depend on the regulation and non-monetary considerations, such as lead time variability.

The impact of carbon footprinting aggregation on realizing emission reduction targets is investigated in the paper by J.C. Velázquez-Martínez, J.C. Fransoo, E.E. Blanco and J.Mora-Vargas. They implement a detailed estimation method and different aggregate approaches for transportation carbon emissions in the dynamic lot sizing model. Analytical results show the limitations of aggregate models for both accurate estimation of real emissions and risks of compliance with carbon constraints. The study provides insights under which conditions aggregate estimations can be used safely and when more detailed estimates are appropriate.

In their paper H.W. Kopfer, J. Schönberger and H. Kopfer propose an approach focusing on the reduction of $\mathrm{CO}_{2}$ emissions caused by transportation. Based on the observation that vehicles with different maximal payload values have different payload-dependent fuel consumption characteristics, the authors introduce the Emission Minimization Vehicle Routing Problem with Vehicle Categories (EVRPVC) which aims at minimizing fuel consumption and $\mathrm{CO}_{2}$ emissions. Comprehensive computational experiments show that considerable fuel savings can be gained by using vehicles of different size.

Minimizing train fuelling cost is the topic of the paper by X. Li, C.-F. Chien, L. Yang and Z. Gao. Since fuel prices vary by location and time, the authors estimate them by use of fuzzy variables and propose a fuzzy optimization model which minimizes the expected fuelling costs under time, speed, and tank capacity constraints. To solve the model, it is decomposed into a nonlinear scheduling and a linear fuelling model. Numerical examples are presented for showing the efficiency of the proposed solution approach.

The final paper in the special issue by $D$. Stindt and R. Sahamie contains a review of research on closed loop supply chain management in the process industry. The authors put a special emphasis on closed loop supply chain (CLSC) management which is a major enabler for sustainability in value creating networks. The contribution aims to describe and analyze the main characteristics of CLSC planning in the process industry and the related publications, in order to determine 
the evolution and gaps of this current research over time and improve our understanding of this issue.

\section{Concluding remarks}

This special issue has greatly benefited from the cooperation among the authors, reviewers, and editors. We would like to express our sincere thanks to the reviewers for their excellent and timely refereeing. Last, but not least, we thank all authors for their contributions which made this special issue possible. 